\title{
Exploring the Moderating Role of Price Consciousness in Pay-What-You-Want Pricing: An Abstract
}

\author{
Rajat Roy, Fazlul Rabbanee, and Piyush Sharma
}

\begin{abstract}
Pay what you want (PWYW) is a unique participative pricing mechanism that has no minimum price set by the seller, and the buyers can pay whatever price they want (including a price of zero) that the seller has to accept without being able to withdraw the offer. Recent research on PWYW focuses only on the direct effects of variables, such as altruism, price consciousness, and reference prices, and ignores their interactions with each other. We address this gap by exploring the moderating influence of price consciousness on the effects of altruism and internal reference price on PWYW pricing decision. We use a field survey with university students in Australia with an ethnic restaurant setting, to show that both altruism and internal reference price have stronger (weaker) effect on the amounts consumers are willing to pay for consumers with lower (higher) levels of price consciousness. Our findings have important implications for both academic researchers and marketing practitioners in this growing research area.
\end{abstract}

R. Roy $(\bowtie) \bullet$ F. Rabbanee $\bullet$ P. Sharma 